\title{
COLOMBIA 2003-2013: ESTRUCTURA Y TENDENCIAS DE LAS EXPORTACIONES
}

\author{
Por: Giovanni E. Reyes ${ }^{1}$ - Sandra Milena Chacón ${ }^{2}$
}

\section{RESUMEN}

Estudiar las principales características mostradas por las exportaciones de productos desde Colombia y los países a los cuales se destinan las mismas, es el objetivo principal de este documento. El argumento fundamental que se sostiene es que los mercados se mantienen relativamente constantes en cuanto a los montos de la demanda de bienes de Colombia, con las modificaciones de destinos que ha implicado Venezuela y la emergencia del papel que juega actualmente China. El artículo incluye una sección sobre crecimiento económico y los desafíos que deben enfrentar los países menos desarrollados, respecto a los nuevos escenarios de comercio internacional que contienen los actuales procesos de globalización.

Palabras clave: comercio internacional, economía de Colombia, comercio y crecimiento económico.

Clasificación JEL: F12, F14, F43.

1. PhD University of Pittsburgh/Harvard; ex Director del Informe de Desarrollo Humano de Naciones Unidas en Venezuela; Profesor Titular, Escuela de Administración, Universidad Colegio Mayor Nuestra Señora del Rosario, Colombia.

2. Master en Negocios Internacionales, fue coordinadora de programa en Proexport, Colombia, actual Directora del Pregrado de Logística, Escuela de Administración de la Universidad Colegio Mayor Nuestra Señora del Rosario, Colombia. 
COLOMBIA 2003-2013: TRENDS AND STRUCTURE OF EXPORTS

\author{
By: Giovanni E. Reyes - Sandra Milena Chacón
}

\begin{abstract}
The main objective of this study is to consider major features belonging to exports from Colombia and countries which are being markets for those goods. The central argument to claim here is that market demand for Colombian exports are keeping relatively unchanged, notwithstanding variations derived from the market links with Venezuela -deterioration- and China -emerging demand. This paper includes conceptual or theoretical considerations regarding new challenges less developed countries need to face in relation to the new conditions or international trade within circumstances of the present globalization phenomena.
\end{abstract}

Key words: international trade, Colombian economy, international commerce and economic growth.

JEL classification: F12, F14, F43. 


\section{Introducción}

El propósito fundamental de este documento es mostrar la situación del comercio exterior de Colombia; en específico, evidenciar la estructura de mercados, su comportamiento en los pasados diez años, además de establecer la concentración de destinos. Entre los aspectos a dilucidar en el estudio está la determinación de que si se han diversificado o no los socios comerciales de Colombia, pero a la vez si ha existido o no concentración de volúmenes de exportación con socios particulares.

En una primera parte se abordan los principales obstáculos que tienen en general los países en desarrollo respecto a su participación en el mercado mundial. En una segunda sección se caracterizan las exportaciones y las exportaciones totales de Colombia en relación con lo que son las variables vitales de la economía de todo país: (i) tasa de crecimiento económico; (ii) inflación o elevación generalizada de precios; y (iii) tasa de desempleo.

Es de tomar en cuenta que para septiembre de 2014, Colombia había firmado ya 12 tratados de libre comercio, entre los que sobresalen los establecidos con Estados Unidos, con Europa, Corea del Sur, Centroamérica, la Comunidad Andina de Naciones -tratado de integración que incluye también a Ecuador, Perú y Bolivia- así como los vínculos con MERCOSUR -integrado en la actualidad, 2014, por Argentina, Brasil, Paraguay, Uruguay y Venezuela-; siendo Chile y Bolivia, países observadores $^{3}$.

En cuanto a la influencia de los tratados de libre comercio debe tomarse en cuenta la estructura de las empresas en Colombia. En términos generales esta estructura está caracterizada por el hecho de que cerca de un 95 por ciento de las empresas, organizaciones o unidades de producción pertenecen a los grupos de micro, pequeñas y mediana empresa. Se estima que de ese porcentaje, un 90 por ciento no llega a estar en operaciones más allá del $7^{\circ}$ año de funcionamiento (Pérez, 2007 y Urdaneta, 2005).

A lo anterior, debe agregarse que una empresa en la medida que se aproxima a la dimensión micro, depende fundamentalmente de la rotación del inventario para poder ser perdurable. En contraste en empresas grandes o muy grandes, la perdurabilidad dependería más de la solvencia -activo fijo dentro del circulante. No obstante, las empresas grandes también pueden tener problemas de liquidez, pero éstos tenderían a ser más bien coyunturales y se pueden superar dado el notable acceso a crédito bancario que estas organizaciones suelen tener (Sender, 2005; Murphy, 2008).

3. Actualizaciones de tratados comerciales en Colombia, en relación con la dinámica del mundo, la influencia de los países asiáticos y la comparación particular con Chile, véase: Carrasquilla, Alberto (2013) “TLC Venteado" en: Dinero, revista de negocios de Colombia, del 9 de abril de 2013; para ampliaciones sobre Comunidad Andina, véase el documento: Comunidad Andina (1969) Acuerdo Básico; Acuerdo de Cartagena. (Lima, Perú: Ediciones CAN), en especial Capítulo II: De la Comunidad Andina y el Sistema Andino de Integración, pp. 3-14; Capítulo Armonización de Políticas Económicas y Planes de Desarrollo, pp. 1-18; sobre el Mercado Común del Sur y las últimas decisiones del Consejo de Presidentes, véase: Mercosur (2014) XLIII Reunión Extraordinaria del Grupo Mercado Común. (Montevideo, Uruguay: Secretaría de Mercosur). 
Los anteriores conceptos se relacionan con los tratados de libre comercio en el sentido de que la competitividad y perdurabilidad de empresas en general está más comprometida, es más vulnerable en la empresa cuando la misma tiende a ser pequeña. Los tratados de libre comercio -en especial el de Estados Unidos y Europa- tenderán a ser una amenaza para las pequeñas y medianas empresas. Un factor que opera en este sentido es el conjunto de políticas de subsidio, cuotas y medidas no arancelarias que establecen las economías más desarrolladas (World Bank, 2010).

\section{Colombia y desafíos de los países en desarrollo frente a las condiciones económicas internacionales}

De conformidad con información dada a conocer por la Comisión Económica para América Latina y el Caribe (CEPAL) se esperaría que, para 2014, Colombia alcanzaría un crecimiento económico entre 4.8 y 5.1 por ciento. Se trataría del mayor crecimiento económico de la región superado tan sólo por Panamá y Bolivia. La perspectiva de incremento del producto interno bruto colombiano para 2014 se situaría por encima del 2.2 por ciento que se espera alcance el promedio latinoamericano (CEPAL, 2014).

La misma CEPAL establece, en relación directa con la estructura de exportaciones colombianas, que la debilidad estructural que se presenta, radica en que se continúa dependiendo de productos relacionados estrechamente con materias primas, tales como petróleo y carbón. El desafío consistiría en que se debe transformar esa naturaleza en las exportaciones propiciando que las mismas lleguen a tener mayor valor agregado. También se impone la necesidad de diversificación de los productos de exportación (CEPAL, 2014; Alfonso y Patiño, 2007).

De conformidad con estudios de mayor tradición y que mantienen la tradición de elaboración teórica de la Comisión Económica para América Latina y el Caribe (CEPAL) y en particular de Ricardo Ffrench-Davis, así como de autores de economía y política internacional, un resumen de los principales desafíos que deben enfrentar se especifica a continuación (French-Davis, 2005; Gilpin, 2008).

a) Heterogeneidad de los mercados externos. Se refiere a que muchas veces se asume que los mercados externos son integrados y estables. Pero otras son las condiciones que por lo general enfrentan las naciones de menor desarrollo relativo: mercados no integrados lo que aumenta la volatilidad en la colocación de las exportaciones y contribuye también a incrementar la vulnerabilidad externa de las naciones de economías más pequeñas.

b) Inestabilidad externa: de manera específica muchos bienes de los países en desarrollo experimentan grandes fluctuaciones en los precios con una tendencia a una baja en los términos de intercambio de estas naciones. Esta situación se transmite al mercado interno a través de repercutir en la balanza de pagos y liquidez interna. Con ello se contribuye a promover una asignación ineficiente de recursos productivos y se generan mecanismos que desembocan en presiones 
inflacionarias. Esto a su vez, tiende a generar una subutilización de la capacidad productiva y fortalece la tendencia al desempleo permanente o estructural ${ }^{4}$.

c) Condicionamiento de los mercados mundiales respecto a negociaciones y factores complementarios. En especial los países de menor desarrollo relativo enfrentan escenarios en los cuales disminuye su capacidad de negociación y de influencia en los mercados internacionales. De allí que la integración y la coordinación en la negociación sean factores que pueden disminuir la dependencia de condicionantes y aumentar la interdependencia. Existen, no obstante, factores complementarios que limitan la capacidad de negociación. Por ejemplo, la deuda externa y las condicionantes de los planes de ajuste, lo que ha limitado la eficacia negociadora de los países con mercados pequeños, tanto en América Latina como en otras regiones de desarrollo.

A manera de resumen, y con base en las tendencias de la negociación aún sobreviviente de la Ronda de Doha o del Desarrollo de la Organización Internacional de Comercio (OIC), es poco probable que Estados Unidos y Europa abran efectivamente sus mercados. Además, Estados Unidos mantiene fuertes subsidios a la producción, sobre todo, agrícola, en particular de alimentos.

Esos elementos promoverán, como ya está ocurriendo en el caso de México, la incapacidad de producción de alimentos por parte de los países de América Latina, de manera que la vulnerabilidad económica externa se verá aumentada ${ }^{5}$.

En todo caso, existen fuertes y persistentes nexos entre los aspectos internacionales, uno de los cuales es el comercio, y el desarrollo de los países. En especial, la influencia del comercio internacional en el crecimiento económico ha sido extensamente estudiada y forma parte del contenido de estudios económicos clásicos en cuanto a: (i) desempeño económico -Smith, Ricardo y Marx; (ii) teoría del valor -Ricardo, Walras y Shumpeter; (iii) relación con sostenibilidad y pleno

4. Los gobiernos tienen básicamente cuatro instrumentos de política para influir en los procesos de industrialización y comercio exterior. Estos cuatro instrumentos son: (i) aranceles; (ii) restricciones cuantitativas al comercio; (iii) varias modalidades de subsidios; y (iv) manejo de la política cambiaria. Los aranceles llevan consigo pérdidas por ineficiencia productiva, lo mismo que las cuotas de importación. Estas condiciones pueden promover una sobreprotección indeseada en algunos sectores, favorecer oligopolios o monopolios y aumentar los costos en los consumidores. No obstante, pueden existir condiciones de carácter político que justifiquen el proteccionismo comercial. En general, los subsidios pueden alcanzar efectos similares en la protección comercial, sin conllevar en demasía, las complicaciones adicionales de otras medidas, tal el caso de cuotas. Véase Perkins, Walter. et. al. (2011). Economics of Development (New York: W.W. Norton Press); Silberberg, Ernest (2008). The Structure of Economics: A Mathematical Analysis (Nueva York: McGraw-Hill), y Hoekman, Harold (2009). The Political Economy of the World Trading System. (Oxford: Oxford University Press).

5. Complementando este aspecto con razones para el proteccionismo, a fin de que los empresarios se comprometan en líneas directamente vinculadas al abastecimiento de mercados locales, se pueden establecer modalidades de protección. Este proteccionismo, en todo caso, debe ser selectivo y debe aplicarse a industrias en desarrollo. Una vez superadas esas fases iniciales el proteccionismo no estaría justificado en términos de eficiencia de producción. De nuevo los subsidios tienen menores efectos de distorsión que otras medidas, especialmente el establecimiento de cuotas. Para ampliaciones: Gilpin, Robert (2008). The World Economy in the 21st. Century (Princeton: Princeton University Press). 
empleo -Keynes, Friedman y Hayek; (iv) ciclos de causación acumulativa y desarrollo -en general la corriente post-keynesiana, Krugman, Myrdal, Stiglitz; y (v) neoinstitucionalisimo -principalmente North ${ }^{6}$.

\section{Variables macroeconómicas y exportaciones colombianas}

Con base en el Cuadro 1, se puede apreciar una caracterización general de las tres variables vitales que un país posee en materia macroeconómica -crecimiento económico, inflación y empleo- además de los datos de exportaciones e importaciones. Las exportaciones tienden a ser mayores que las importaciones, de conformidad con las cifras de media aritmética, mediana así como los valores máximos.

El valor mayor de la desviación estándar de las exportaciones indica que esta variable tiene mayor volatilidad que las importaciones, aun cuando los valores de los coeficientes de variación tienden a mostrar diferencias matemáticas, más no estadísticas.

La variable de empleo, durante al menos los pasados ocho años, ha sido preocupante en la economía colombiana. Colombia es, junto con Uruguay, Panamá y Perú, de los países que menos han sido impactados por la última crisis económica financiera internacional. La crisis tuvo su punto de inflexión el 13 de septiembre de 2008 con la quiebra del banco de inversión Lehman Brothers.

Uno de los postulados o articulaciones más importantes consiste en que, para que el crecimiento económico tenga una repercusión positiva en el potencial de desarrollo, debe promover esencialmente la creación de puestos de trabajo. En la medida que se incrementen los empleos en la economía real -la que se relaciona con la producción de bienes, servicios y empleo- se ven aumentadas las oportunidades para la población (Hull, 2009; Gutiérrez, 2007).

Los Cuadros 2, 3 y 4 muestran las principales características de las exportaciones colombianas en función del volumen o quantum de las mismas, la participación porcentual -incluso discriminando entre el peso de los 10 primeros destinos de producto y otras plazas- los pesos porcentuales y los puntajes de Z de los principales compradores de productos de Colombia.

6. Dentro de las obras y los comentarios, ensayos o actualizaciones más representativas: Smith, Adam (2004) Teoría de los Sentimientos Morales. (México, D.F., México: Fondo de Cultura Económica); Smith, Adam \& Hayek, Friedrich (2007) Ética y Economía. (México, D.F., México: Editorial de la Universidad Iberoamericana); Ricardo, David (2007) Ricardo's High Price of Bullions and an Essay on Profits. (Nueva York: Cosimo Publs.); Arestis, Philip (2004) Crítica a la Economía Ortodoxa. (Barcelona, España: Universidad Autónoma de Barcelona); Krugman, Paul (2007) Rethinking International Trade. (Massachusetts: MIT Press); Myrdal, Gunnar (2004) Asian Drama: An Inquiry into the Poverty of Nations. (Nueva Delhi: India: Kalyani Publishers); Stiglitz, Joseph (2008) Los Felices Noventa y la Semilla de la Destrucción. (Bogotá, Colombia: Taurus); Stiglitz, Joseph (2002) El Malestar con la Globalización. (Bogotá, Colombia: Taurus); North, Douglass. (1981). Structure and Change in Economic History. (New York: W. W. Norton \& Co.); y North, Douglass. (2008). Institutions, Institutional Change and Economic Performance. (New York: Cambridge University Press). 


\section{Cuadro 1}

Colombia 2000-2013: crecimiento económico y variables macroeconómicas (estadísticos descriptivos)

\begin{tabular}{lccccc}
\hline \multicolumn{1}{c}{ Indicador } & $\begin{array}{c}\text { Crecimiento } \\
\text { Económico }\end{array}$ & Exportación & Importación & Inflación & Desempleo \\
\hline Media aritmética & 4.08 & 4770 & 3409 & 5.08 & 13.87 \\
Mediana & 4.15 & 3975 & 2678 & 5.20 & 13.10 \\
Máximo & 7.50 & 12439 & 7340 & 8.80 & 18.10 \\
Mínimo & 0.30 & 2298 & 1706 & 2.10 & 10.20 \\
Rango & 7.20 & 10141 & 5634 & 6.70 & 7.90 \\
Desv. Estándar/1 & 1.91 & 2489 & 1822 & 2.10 & 2.77 \\
Coef. de Variac. ${ }^{2}$ & 0.46 & 0.52 & 0.53 & 0.41 & 0.19 \\
\hline
\end{tabular}

Notas: 1/ desviación estándar de la muestra, esto es con el coeficiente de corrección de Pearson en el denominador $(n-1)$;

2/ Coeficiente de variación de conformidad con: $\left\{\left[(\text { Desv. Est. / Med. Aritmética) }]^{*} 100\right\}\right.$.

Fuente: Cálculos de este estudio con base en cifras de Proexport, Colombia.

\section{Cuadro 2}

Colombia 2003: exportaciones totales (US\$ corrientes)

\begin{tabular}{lccc}
\hline \multicolumn{1}{c}{$\begin{array}{c}\text { País de Destino } \\
\mathbf{2 0 0 3}\end{array}$} & Millones de US\$ & $\begin{array}{c}\text { Participación } \\
\%\end{array}$ & $\begin{array}{c}\text { Puntaje } \\
\mathbf{Z}^{1{ }^{11}}\end{array}$ \\
\hline Estados Unidos & 5779 & 44.0 & 2.818 \\
Ecuador & 780 & 5.9 & -0.047 \\
Venezuela & 696 & 5.3 & -0.095 \\
Perú & 369 & 3.0 & -0.267 \\
Países Bajos & 302 & 2.3 & -0.321 \\
Chile & 189 & 1.4 & -0.386 \\
Panamá & 172 & 1.3 & -0.395 \\
Suiza & 129 & 1.0 & -0.420 \\
Brasil & 93 & 0.7 & -0.440 \\
China & 83 & 0.6 & -0.447 \\
\hline Sub-Total Top 10 & 8627 & 65.7 & \\
Otros & 4500 & 34.3 & \\
Total General & 13127 & 100.0 & \\
No. de Mercados & 180 & & \\
No. de Empresas & 9881 & & \\
Promedio Top & & 6.57 & \\
Desviación Estándar ${ }^{2 /}$ & & 13.29 & \\
\hline
\end{tabular}

Notas: $1 /$ Coeficiente $Z$, con base en [(dato $x$ - media aritmética) / desviación estándar $(n-1)$ ].

2/ desviación estándar de la muestra, esto es, con el coeficiente de corrección de Pearson en el denominador $(n-1)$.

Fuente: Cálculos de este estudio con base en cifras de Proexport, Colombia. 
Conforme las cifras del Cuadro 2, los tres principales destinos de exportación, responsables de la compra del 54 por ciento de las exportaciones colombianas, eran Estados Unidos, Ecuador y Venezuela. Nótese sin embargo el gran peso del mercado estadounidense lo que se hace evidente al tener este país el único dato positivo en los puntajes de Z.

Conforme al Cuadro 3, los mercados de los productos colombianos demuestran un cambio respecto a 2003. Es ahora Venezuela el segundo destino de las exportaciones y juntos los dos países suman 53 por ciento de las exportaciones. El peso que se incrementó del mercado venezolano se pone de manifiesto en que, junto con Estados Unidos, son los dos países que como socios comerciales tienen valores positivos en la prueba de Z.

\section{Cuadro 3}

COLOMBIA 2008: exportaciones totales (US\$ corrientes)

\begin{tabular}{lccc}
\hline $\begin{array}{c}\text { País de Destino } \\
\mathbf{2 0 0 8}\end{array}$ & Millones de US\$ & $\begin{array}{c}\text { Participación } \\
\text { \% }\end{array}$ & $\begin{array}{c}\text { Puntaje } \\
\mathbf{Z}^{1 /}\end{array}$ \\
\hline Estados Unidos & 18,053 & 37.3 & 2.620 \\
Venezuela & 6,092 & 16.2 & 0.791 \\
Ecuador & 1,500 & 4.0 & -0.263 \\
Suiza & 945 & 2.5 & -0.390 \\
Perú & 855 & 2.3 & -0.411 \\
Chile & 849 & 2.3 & -0.412 \\
Países Bajos & 752 & 2.0 & -0.434 \\
Brasil & 649 & 1.7 & -0.458 \\
China & 443 & 1.2 & -0.505 \\
Panamá & 319 & 0.8 & -0.534 \\
\hline Sub-Total Top 10 & 26,814 & 71.3 & \\
Otros & 10,812 & 28.7 & \\
Total General & 37,626 & 100.0 & \\
No. de Mercados & 205 & & \\
No. de Empresas & 11,191 & & \\
Promedio Top & & 7.03 & \\
Desviación Estándar ${ }^{2 /}$ & & 11.57 & \\
\hline
\end{tabular}

Notas: $1 /$ Coeficiente $Z$, con base en [(dato $x$ - media aritmética) / desviación estándar ${ }_{(n-1)}$ ].

$2 /$ desviación estándar de la muestra, esto es, con el coeficiente de corrección de Pearson en el denominador $(n-1)$.

Fuente: Cálculos de este estudio con base en cifras de Proexport, Colombia.

El contenido del Cuadro 4 contiene ya los efectos de la crisis financiera mundial cuyo punto de inflexión fue la quiebra del banco de inversiones Lehman Brothers. Se tienen aquí también, los impactos producto de los problemas políticos y de 
liderazgo entre los presidentes de Colombia y Venezuela -Álvaro Uribe Vélez y Hugo Chávez, respectivamente-. Nótese cómo Venezuela pasó de ser un mercado para Colombia que tenía un peso de 16 por ciento de las exportaciones, en 2008, a un poco menos de 4 por ciento en 2013.

Por otra parte, es notorio el posicionamiento de China que, conforme lo datos más actuales, es el segundo destino de los productos de Colombia, y junto a Estados Unidos tiene para 2013 un puntaje positivo de Z. Es decir que se ubican más allá del promedio aritmético.

\section{Cuadro 4}

Colombia 2013: exportaciones totales (US\$ corrientes)

\begin{tabular}{lccc}
\hline \multicolumn{1}{c}{$\begin{array}{c}\text { País de Destino } \\
\mathbf{2 0 1 3}\end{array}$} & Millones de US\$ & $\begin{array}{c}\text { Participación } \\
\%\end{array}$ & $\begin{array}{c}\text { Puntaje } \\
\mathbf{Z}^{\prime \prime}\end{array}$ \\
\hline Estados Unidos & 18,459 & 31.4 & 2.763 \\
China & 5,102 & 8.7 & 0.240 \\
Panamá & 3,329 & 5.7 & -0.094 \\
Países Bajos & 2,266 & 3.9 & -0.295 \\
Venezuela & 2,256 & 3.8 & -0.297 \\
Ecuador & 1,975 & 3.4 & -0.350 \\
Brasil & 1,591 & 2.7 & -0.422 \\
Chile & 1,572 & 2.7 & -0.426 \\
Perú & 1,274 & 2,2 & -0.482 \\
Suiza & 461 & 0.8 & -0.636 \\
\hline Sub-Total Top 10 & 38,283 & 65.1 & \\
Otros & 20,539 & 34.9 & \\
Total General & 58,822 & 100.0 & \\
No. de Mercados & 204 & & \\
No. de Empresas & 9,989 & & \\
Promedio Top & & 6.51 & \\
Desviación Estándar ${ }^{2 /}$ & & 9.00 & \\
\hline
\end{tabular}

Notas: $1 /$ Coeficiente $Z$, con base en [(dato $x$-media aritmética) / desviación estándar ${ }_{(n-1)}$ ].

2 / desviación estándar de la muestra, esto es, con el coeficiente de corrección de Pearson en el denominador $(n-1)$.

Fuente: Cálculos de este estudio con base en cifras de Proexport, Colombia.

Siguiendo este mismo indicador, que la economía estadounidense sea el mercado principal de la producción colombiana se confirma con más de 2 de coeficiente de Z, lo que -de conformidad con un fenómeno de distribución normal, siendo Z una prueba paramétrica- estaría superando el 96 por ciento de las probabilidades estadísticas de influencia en el fenómeno bajo estudio. 
El Cuadro 5 sintetiza las comparaciones a partir de los datos contenidos en los cuadros 2, 3 y 4. Es de resaltar que Estados Unidos no sólo es el principal destino de los productos de exportación de Colombia, sino que además se tiende a consolidar en el período entre 2003 y 2013. La diferencia positiva, esto es el incremento de las exportaciones a este mercado, creció en 12,680 millones de dólares.

\section{Cuadro 5}

Colombia 2013 - 2013: Datos comparativos y tendencias de exportaciones totales (US\$)

\begin{tabular}{lccc}
\hline \multicolumn{1}{c}{ País de Destino } & $\begin{array}{c}\text { Diferencia 2013-2003 } \\
\text { en Millones de US\$ }\end{array}$ & $\begin{array}{c}\text { Participación } \\
\%\end{array}$ & $\begin{array}{c}\text { Coeficientes de } \\
\text { Correlación }\end{array}$ \\
\hline Estados Unidos & 12,680 & 319 & 0.493 \\
China & 5.020 & 6,177 & $0.880^{*}$ \\
Panamá & 3,156 & 1,931 & $0.846^{*}$ \\
Países Bajos & 1,964 & 750 & $0.941^{* *}$ \\
Venezuela & 1,560 & 324 & 0.122 \\
Ecuador & 1.195 & 253 & $0.938^{* *}$ \\
Brasil & 1,497 & 1,703 & $0.936^{* *}$ \\
Chile & 1,383 & 833 & $0.863^{*}$ \\
Perú & 878 & 322 & $0.930^{* *}$ \\
Suiza & 332 & 357 & 0.608 \\
\hline Sub-Total Top 10 & 29,656 & 65 & \\
Otros & 16,038 & 35 & \\
Total General & 45,694 & 100.0 & \\
No. de Mercados & 24 & 113 & \\
No. de Empresas & 108 & 101 & \\
Promedio Top & & 6.57 & \\
Desviación Estándar ${ }^{2 /}$ & & 13.29 & \\
\hline
\end{tabular}

Notas: $1 /$ Coeficiente de correlación de Pearson ${ }^{7}$;

2/ desviación estándar de la muestra, esto es, con el coeficiente de corrección de Pearson en el denominador $(\mathrm{n}-1)$.

Fuente: Cálculos de este estudio con base en cifras de Proexport, Colombia.

7. Coeficiente de correlación de Pearson, con base en covarianzas y desviaciones típicas de "x" y "y", como variables independientes y de frecuencias:

$$
r_{x y}=\frac{\sum x_{i} y_{i}-n \bar{x} \bar{y}}{n s_{x} s_{y}}=\frac{n \sum x_{i} y_{i}-\sum x_{i} \sum y_{i}}{\sqrt{n \sum x_{i}^{2}-\left(\sum x_{i}\right)^{2}} \sqrt{n \sum y_{i}^{2}-\left(\sum y_{i}\right)^{2}}} .
$$


No obstante lo anterior, es también importante subrayar que China y Panamá se logran posicionar como mercados importantes. Esto último se pone de manifiesto no solamente con el posicionamiento directo, sino también con los coeficientes de correlación que se obtuvieron de la serie en estudio. En este sentido, las tendencias más consistentes al alza corresponden a China, Panamá, Países Bajos, Brasil, Chile y Perú.

En una situación contrastante, en relación con los anteriores países, se encuentra Venezuela. En este caso el coeficiente de correlación lineal de la serie llegó a tan sólo 0.122 .

Con ello se tiene evidencia que el comportamiento es muy variable, que no es sostenido en el tiempo, ya sea para declinar o para aumentar participación. De nuevo, esto es un indicador de las dificultades políticas surgidas especialmente en el segundo período de gobierno del Presidente Álvaro Uribe con el Presidente Hugo Chávez (2006-2010).

\section{Conclusiones y consideraciones finales}

Los destinos de los productos colombianos no manifiestan una mayor diversificación en cuanto a expansión de países de destino. Se concentran en un número relativamente reducido de países.

Estados Unidos continúa siendo el principal comprador y su presencia como tal se mantiene en una tercera parte del destino de exportaciones colombianas. Lo que sí ha mostrado variaciones es el conjunto de otros países que complementan en segunda, tercera y cuarta posiciones los mercados de productos de Colombia. En este último sentido se han posicionado China, Panamá y Países Bajos.

El aumento total de exportaciones de Colombia en el período 2003 a 2013 fue de 45,694 millones de dólares. En esto se tuvo mucha influencia, más que del quantum de las exportaciones, del precio de los mercados internacionales, los que mostraron un notable repunte, en especial durante el período 2003 a 2009.

Venezuela es el caso más llamativo en cuanto a perder su posición de ser el segundo socio comercial tradicional de Colombia y ser superado por otros países, en particular por China. Complementariamente, el número de empresas que se ha incorporado a la exportación en el período bajo estudio ha sido relativamente pequeño; ellas fueron 108, para completar un total de 9,989 -un incremento de 1.08 por ciento-. 


\section{REFERENCIAS}

ABONYI, Janos (2007). Cluster Analysis for Data Mining and Systemic Identification (Basel, Germany: Pannonia).

ALCAIDE, Carlos (2010). Fidelización de Clientes. (Madrid, España: ESIC).

ALFONSO, José y Patiño, Carlos (2007). ¿Crecer para exportar o exportar para crecer? El caso del Valle del Cauca (Cali, Colombia: Centro Regional de estudios Económicos de Cali; documento No. 46).

ANDERSON, David; et. al. (2003). Estadística para Administración y Economía (México, D.F.: Thompson Editores).

ARESTIS, Philip (2004). Crítica a la Economía Ortodoxa (Barcelona, España: Universidad Autónoma de Barcelona).

BANCO DE LA REPÚBLICA DE COLOMBIA (2003) Comercio Exterior y Actividad Económica de Colombia (Bogotá, Colombia: Banco de la República, Serie de Publicaciones, Borradores de Economía No. 163; http://www.banrep.gov.co/es/borrador163).

CANALES, Manuel (2008). Metodologías de la Investigación Social (Santiago de Chile: LOM, Ediciones).

CARRASQUILLA, Alberto (2013). "TLC Venteado" en: Dinero, revista de negocios de Colombia, del 9 de abril de 2013.

COMISIÓN ECONÓMICA PARA AMÉRICA LATINA Y EL CARIBE (CEPAL) (2014). Estudio Económico de América Latina y el Caribe 2014 (Santiago de Chile: CEPAL).

COMUNIDAD ANDINA (1969). Acuerdo Básico; Acuerdo de Cartagena (Lima, Perú: Ediciones CAN).

EVERITT, Brian, et. al. (2011). Cluster Analysis: Probability and Statistics (New York: Wiley).

FRENCH-DAVIS, Ricardo (2005). Macroeconomía, Comercio y Finanzas para Reformar las Reformas en América Latina (Santiago de Chile: McGraw-Hill-CEPAL).

GARCÍA, Luis (2010). Econometría de Evaluación de Impacto (Lima, Perú: Pontificia Universidad Católica del Perú).

GILPIN, Robert (2008). Global Political Economy (Princeton: Princeton University Press).

GUTIERREZ, C. et al. (2007). “Does Employment Generation Really Matter for Poverty Reduction?”, World Bank Policy Research Working Paper No. 4432, World Bank, Washington D.C.

HULL, K. (2006)."An Overview of World Bank Analytical and Advisory Activities addressing the Labour Market and Employment, 2004-Present", Mimeo, World Bank, Washington D.C.

KEE, Hiau Looi, Alessandro Nicita and Marcelo Olarreaga (2008). Import demand elasticities and trade distortions. The Review of Economics and Statistics. 90(4): 666-682.

KRUGMAN, Paul (2007). Rethinking International Trade (Massachusetts: MIT Press).

LEVIN, Jack (2008). Introducción a Estadística en Investigación Social (México, D.F., México: Harla).

MERCOSUR (2014). XLIII Reunión Extraordinaria del Grupo Mercado Común (Montevideo, Uruguay: Secretaría de MERCOSUR).

MURPHY, A. and E. Strobl (2008). “Employer and Employee Ignorance in Developing Countries: The Case of Trinidad and Tobago," Review of Development Economics, 12(2):339-353, Wiley Blackwell. 
MYRDAL, Gunnar (2004). Asian Drama: An Inquiry into the Poverty of Nations (Nueva Delhi: India: Kalyani Publishers).

NICITA, Alessandro (2013). Exchange Rates, International Trade and Trade Policies (Geneva, Switzerland: United Nations Conference on Trade and Development, UNCTAD).

NORTH, Douglass (1981). Structure and Change in Economic History (New York: W. W. Norton \& Co.).

NORTH, Douglass (2008). Institutions, Institutional Change and Economic Performance (New York: Cambridge University Press).

PÉREZ, Rafael (2007). en el artículo Estructura y Cultura Organizacional en la PYME Colombiana: Análisis en Empresas Bogotanas, en Cuadernos de Administración, Universidad del Valle, No. 38, junio a diciembre.

RAJAN, RAGHURAM \& SUBRAMANIAN, Arvind (2011). Aid, Dutch Disease, and Manufacturing Growth. Journal of Development Economics. 94(1): 106-118.

RICARDO, David (2007). Ricardo 's High Price of Bullions and an Essay on Profits (Nueva York: Cosimo Publs.).

RODRIK, Dani (2008). The Real Exchange Rate and Economic Growth (New York: Brookings Papers on Economic Activity).

ROMESBURG, Charles (2004). Cluster Analysis for Researchers (North Carolina, United States: Wadsworth Publs).

SENDER, J., C CRAMER and C. Oya (2005). Unequal Prospects: Disparities in the Quantity and Quality of Labour Supply in sub-Saharan Africa, World Bank Social Protection Discussion Paper No. 0525, World Bank, Washington, D.C.

SMITH, Adam \& Hayek, Friedrich (2007). Ética y Economía (México, D.F., México: Editorial de la Universidad Iberoamericana).

SMITH, Adam (2004). Teoría de los Sentimientos Morales (México, D.F., México: Fondo de Cultura Económica).

STIGLITZ, Joseph (2002). El Malestar con la Globalización (Bogotá, Colombia: Taurus).

STIGLITZ, Joseph (2008). Los Felices Noventa y la Semilla de la Destrucción (Bogotá, Colombia: Taurus).

URDANETA, Oscar (2005). Psicología Organizacional aplicada a la gestión del Capital Humano (Bogotá, Colombia: 3R Editores).

WORLD BANK (2005). Pro-Poor Growth in the 1990's: Lessons and Insights from 14 Countries, Agence Française pour le Développement, Paris. 